\title{
دراسة تطبيقية لاشاكل صفوف الانتظار للمركبات في بعض همطات التعبئة لمدينة بغداد
}

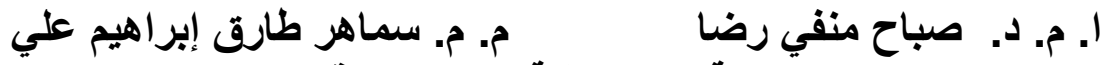 \\ جامعة بغادـ كلية الادارة والاقتصاد \\ وحدة البحوث الإدارية والاقتصادية \\ قسم الاحصاء
}

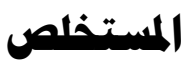

نظراً للظروف التي مر بها قطرنا الحبيب والتي أدت إلى وقوع العليد من الأزمات التي من أهمها أزمة التهة

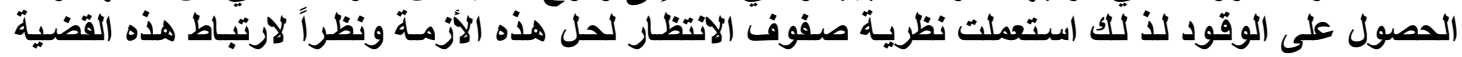

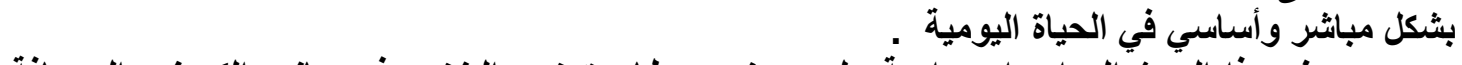

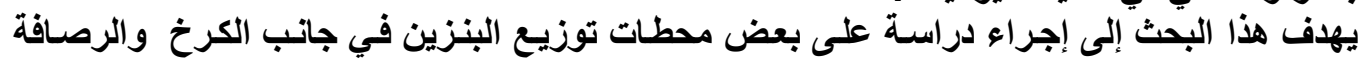

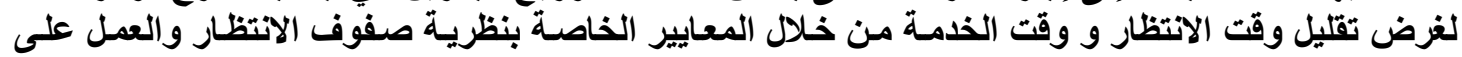

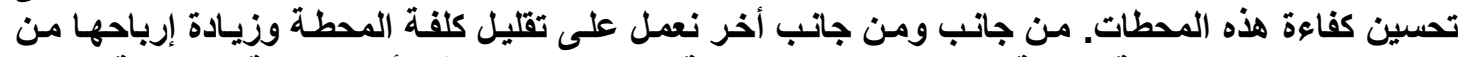

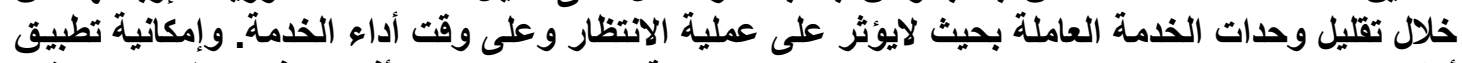

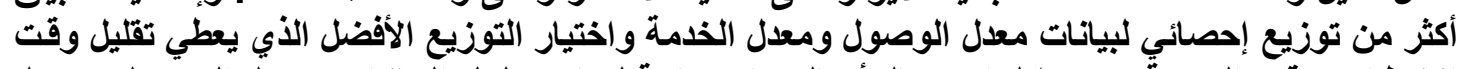

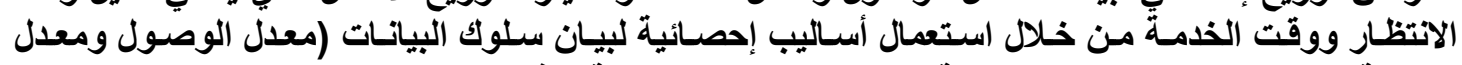

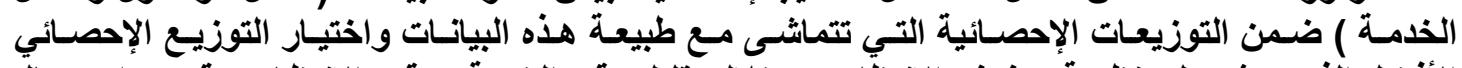

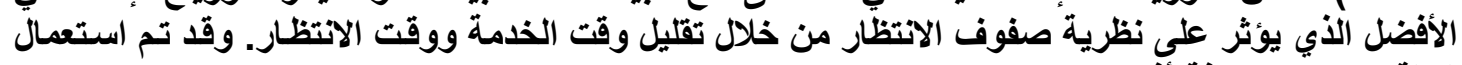
ثلاثة مجاميع لمعرفة أفضل التوزيعات هي لأني

$$
\begin{aligned}
& \text { 1. . بواسون مع أسي } \\
& \text { rا. بواسون مع وييل } \\
& \text { r. ايرلا بلك مع أسي }
\end{aligned}
$$

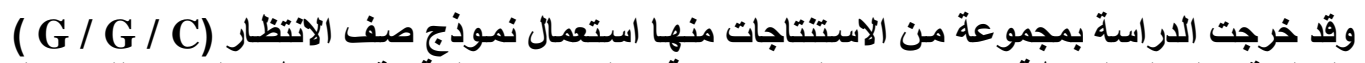

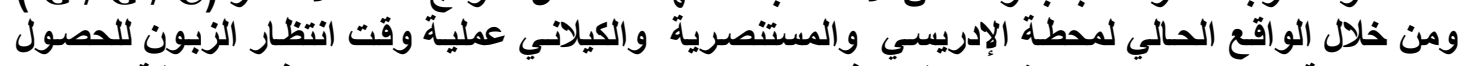

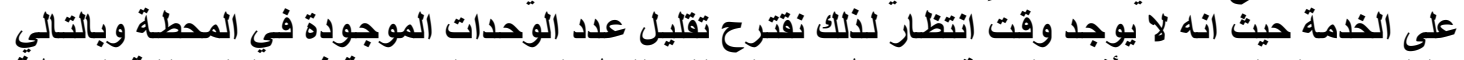

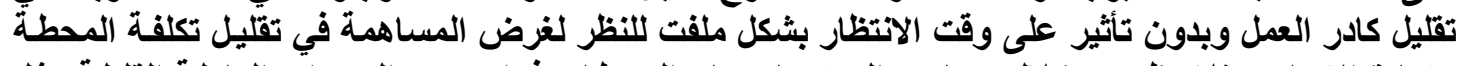

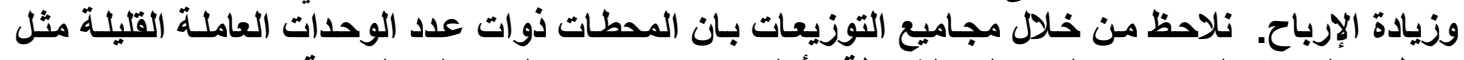

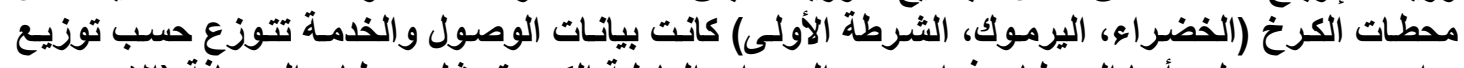

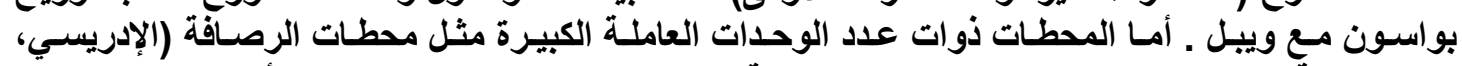

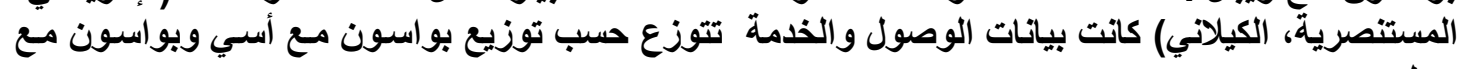




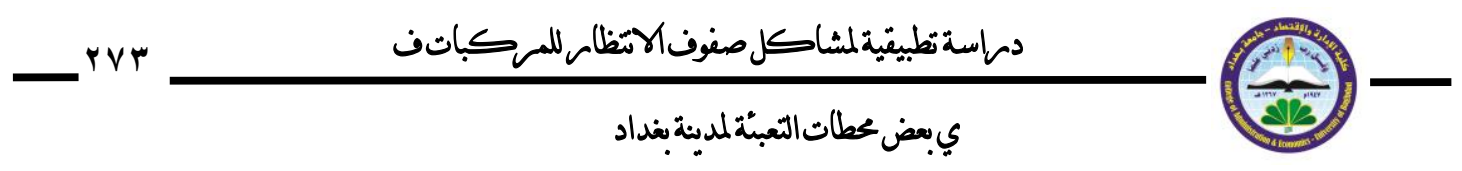

\section{ABSTRACT}

According to the circumstances experienced by our country which led to Occurrence of many crises that are the most important crisis is gaining fuel therefore, the theory of queue ( waiting line ) had been used to solve this crisis and as the relevance of this issue indirect and essential role in daily life .

This research aims to conduct a study of the distribution of gasoline station in (both sides $A L$ - kharkh and AL Rusafa, for the purpose of reducing wasting time and services time through the criteria of the theory of queues and work to improve the efficiency of these stations by the other hand. we are working to reduce the cost of station and increase profits by reducing the active service unit so that do sent affect the process of waiting and the time of performance services. the possibility of applying more than statistical distribution of data acess rate and the rate of services and best selection of distribution which reducing the time of service through statistical techniques to demonstrate the behavior of the data (access rate and service rate) with in the statistical distribution that are consistent with the nature of data and select the best statistical distribution which affects the theory of the queues during the time of service and waiting time there are three categories for better know ledge of distribution are
1. Poisson with Exponential
2. Poisson with Weibull
3. Erlang with Exponential

Asset of on clusion had been emerged from the study as using such (G /G /C) queue model which includes the use through the current reality stations like AL-idrisi, AL- Mustansiriya and AL-galani the waiting time for the customer of services is mostly doesn't have time to wait therefore, we suggest reducing the number of units in station and increase profit through the groups note that the distribution stations animate the few number of unit operating station such as AL- karkh (AL- khadra, AL- yarmouk and AL- shorta al-ola) the data access and service distributed according to the distribution of Poisson with Weibull the data access Service and distributed according to Poisson With Weibull station do the large number of units operating station such as AL- Rusafa (AL-idrisi, AL-Mustansiriya and AL-galani ) the data access and service distribution according to the distribution of Poisson with Exponential and Poisson with Weibull . 


\section{الاقدهة ( Introduction )}

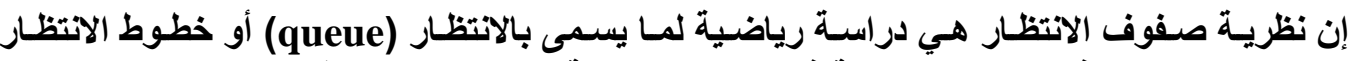
(waiting lines)

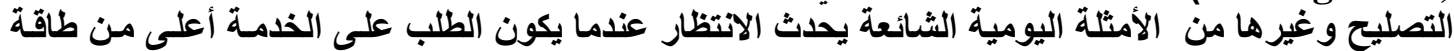

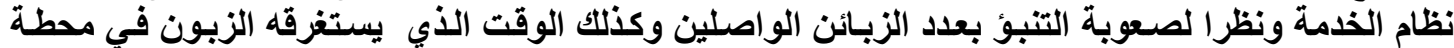

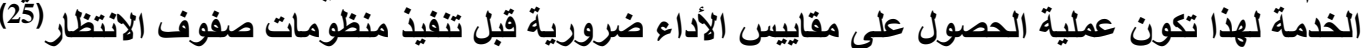

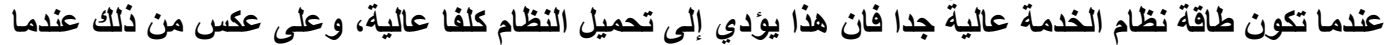

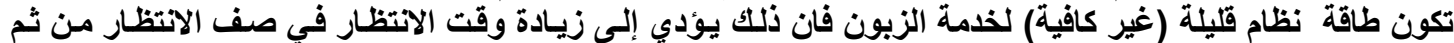

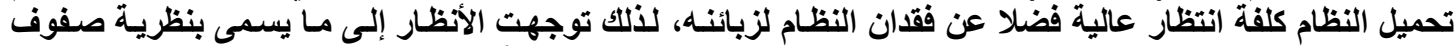

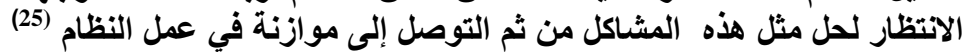

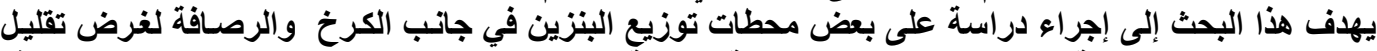

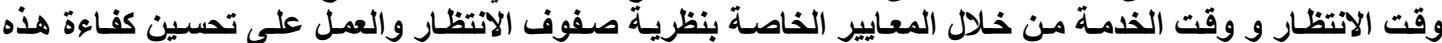

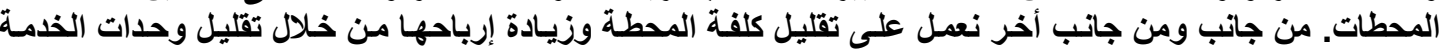

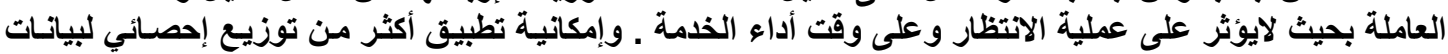

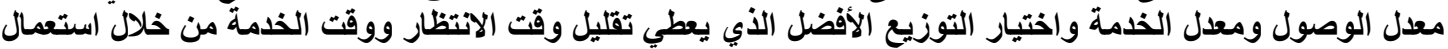

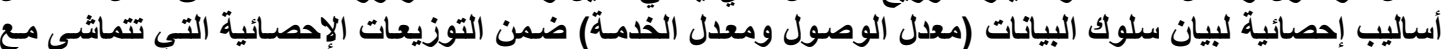

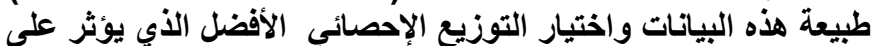
نظرية صفوف الانتظار من خلال تقليل وقت التخدمة و وقت الانتظار. وقد تم تطبيق أكثر من توزيع إحصائي لبيانات

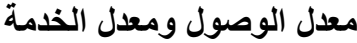

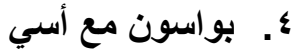

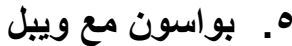

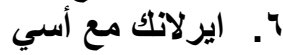

البانب النظري

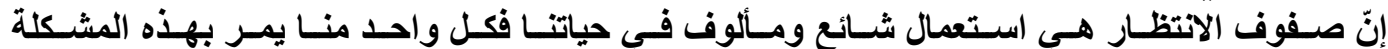

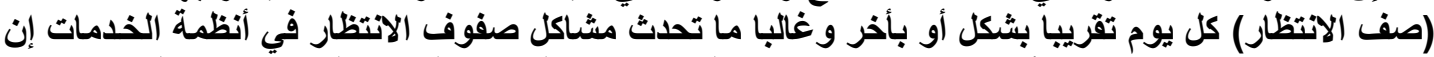

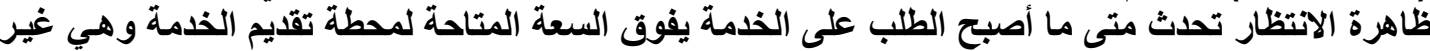

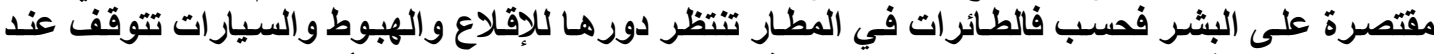
إشارات المرور فضلا عن خطوط الهاتف المزدحمة التي تعد مثالا لثبكات خطوط أو صلئر صفوف الانتظار المعقدة

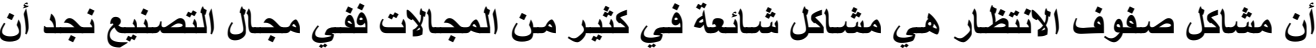

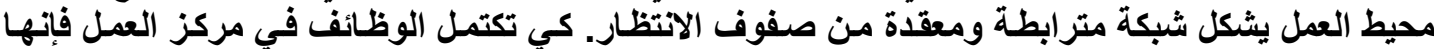

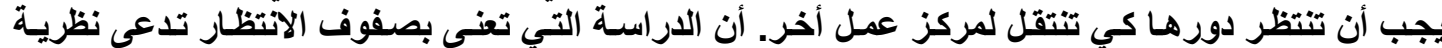
صفوف الانتظار (Queuing Theory) وتتضمن نظرية صفوف الانتظار دراسة رياضية لصفوف الانتظار

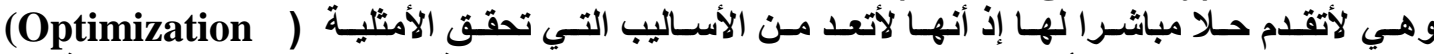
Technique

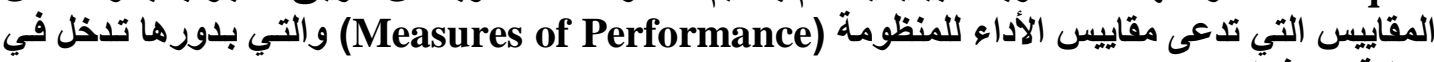
عملية اتخاذ الثقرار.

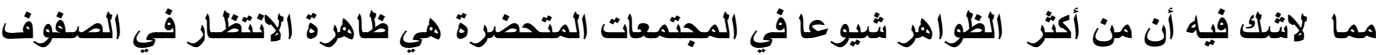

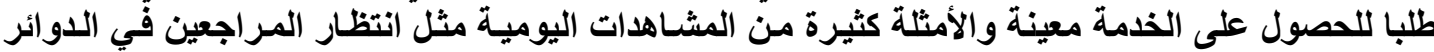

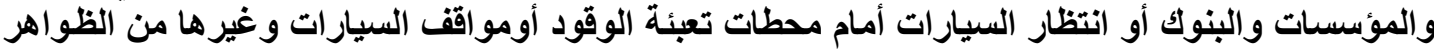

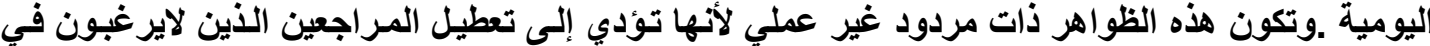

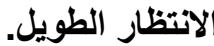




\section{$\underline{\text { Poisson Distribution }}$}

$$
\text { ا ـ ـ الاعتماد في هذا البحث التوزيعات التالية }
$$

أن التوزيع الاحتمـالي المتقطع والمعروف بتوزيع بواسون يستعمل لوصف وصول الزبـائن العشوائي لنظام صف الانتظار إذا تحققت الثروط الآتية :

• الترتيب (Order liness ) : يقصد بـه في أيسة فترة زمنيـة يصل على الأغلب زبون واحد إلى محطة الخدمة . الاستقرارية (Stationarity) ) : أي إنـه ضمن إطـار زمنسي معين تكون احتمالية وصـول زبون

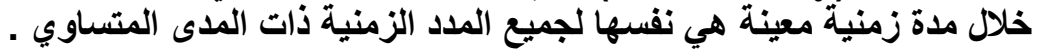

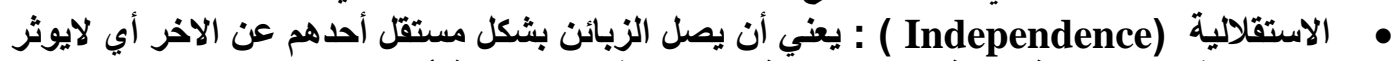

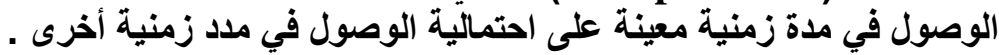

فإذا توافرت الثروط أعلاه في نموذج صف الانتظار أمكن التعبير عن احتمالية ( n) من الواصلين في مدة زمنية بطول أو مدى (t) بالصيغة الآنية

$$
P_{(n)}(t)=\frac{(\lambda t)^{n} e^{-\lambda t}}{n !}, n=0,1,2, \ldots . .
$$

ג : معدل الوصول لكل وحدة زمنية.

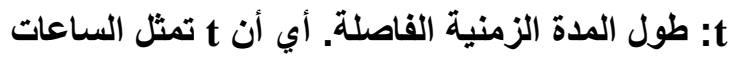

Exponential Distribution

$$
\text { r - ب - التوزيع ألأسي }
$$

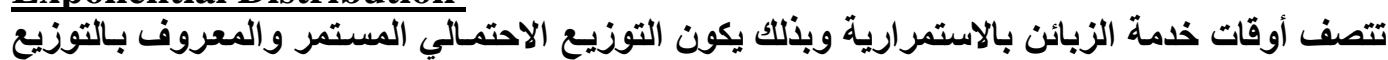

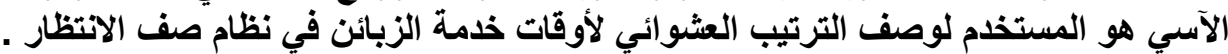

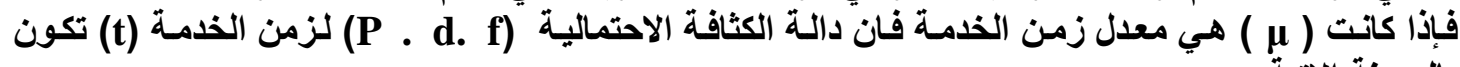
$F(t)=\lambda e^{-\lambda t} \quad, t>0$

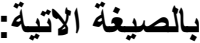
حيث إن:

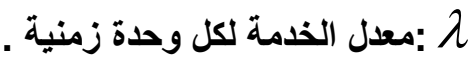
t: بول المدة الزمنية الفاصلة. 


\section{Weibull Distribution}

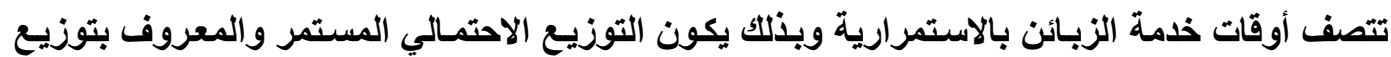

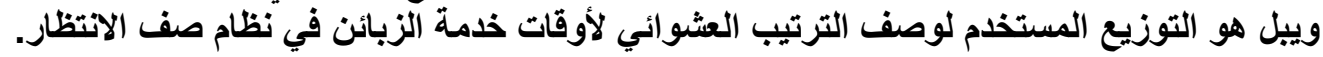
وان الدالة الاحتمالية له (P.d ( ) هي

$$
f(x)=\frac{\beta}{\lambda^{\beta}} x^{\beta-1} e^{-(x / \lambda)^{\beta}} \quad x \geq 0
$$

\section{Generalized Erlang Distribution $\left(\mathbf{G} \mathbf{E}_{\mathbf{k}}\right)$}

$$
\text { ع - توزيع ايرلانك العام }
$$

لقد بينا سابقا انه عندما يكون معامل الاختلاف مسـاوي إلى الواحد

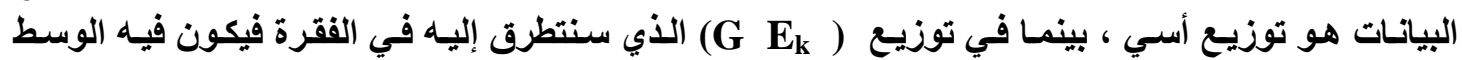

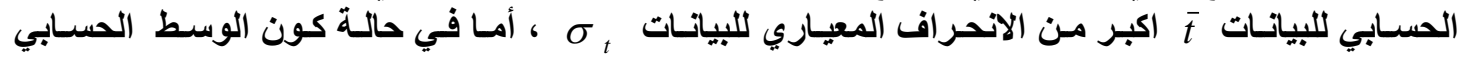
للبيانات $\bar{t}$ هو اقل من الانحراف المعياري للبيانات سوف يسلك سلوكا أخر. ألان لفهم توزيع ايرلاتلك العام بصورة أفضل سوف توفيع نأخذ مخطط الوصول ( 5 - 2 ) والموضح أدناه

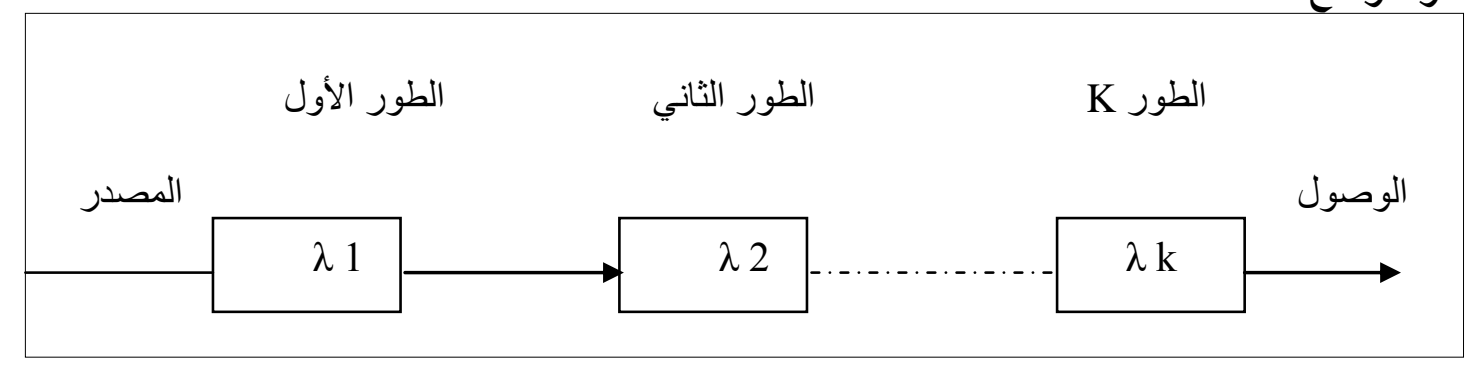

مخطط (5 - 2 ) محطة وصول تتضمن K من الأطوار

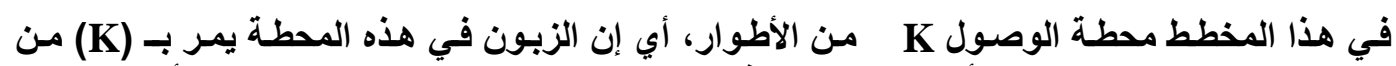

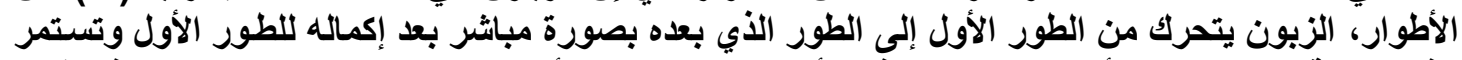

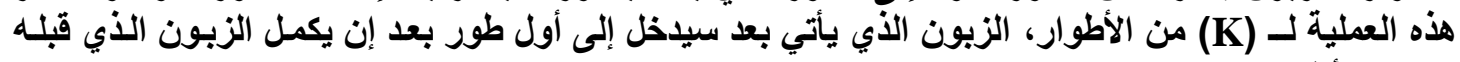

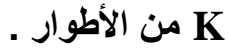

ومن الجدير بالذكر أن القاعدة الأساسية لطريقة الأطوار تعتمد على الخاصية المار كوفية للتوزيع ألأسي .

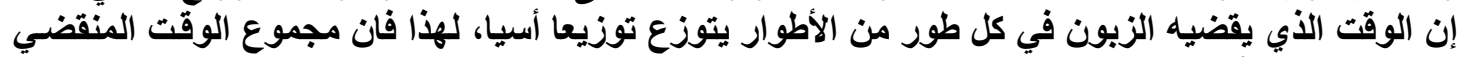

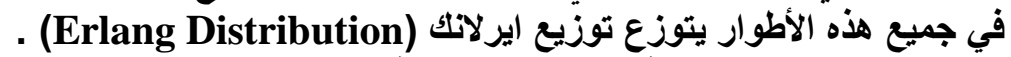

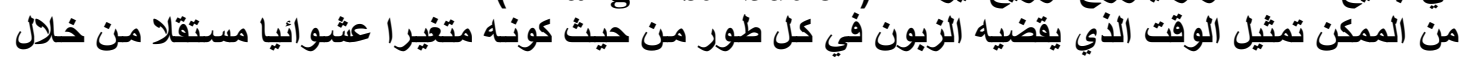
$T_{k}, \quad k=1,2, \ldots \ldots \ldots \ldots \ldots$

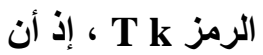

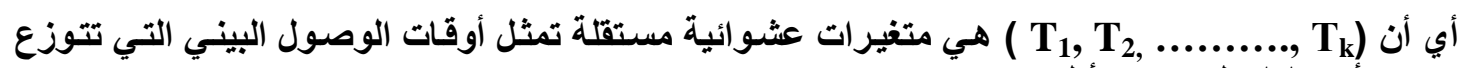
$a_{k}(t), \forall{ }_{k}=1,2, \ldots \ldots \ldots \ldots \ldots$ 


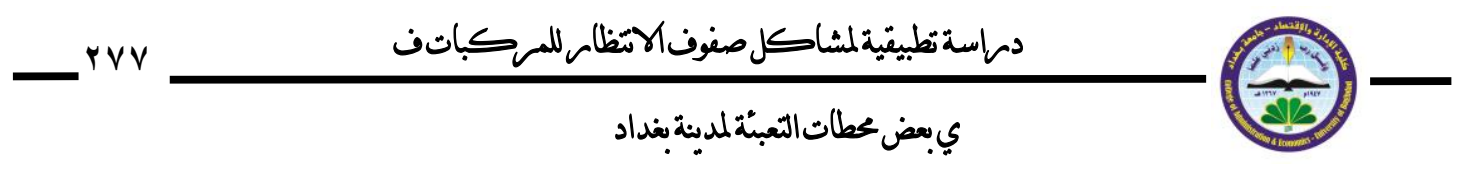

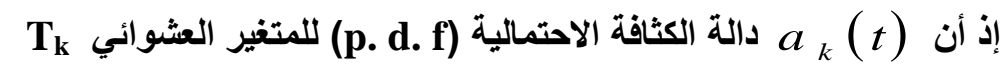
$L_{k}(s), \forall_{k}=1,2$

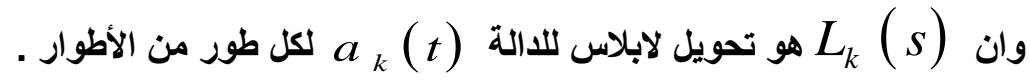

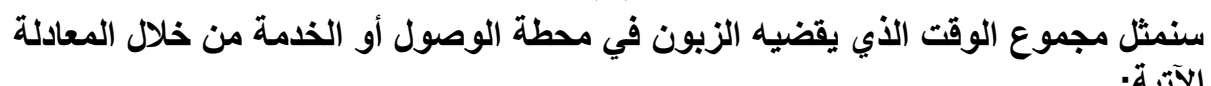

$T=\sum_{k=1}^{K} T_{k}$

$(2-1)$

من معادلة دالة الكثافة الاحتمالية للتوزيع الآسي ، نحصل على :

$a_{k}(t)=\lambda_{k} e^{-\lambda_{k} t} \quad, \forall{ }_{k}=1,2, \ldots \ldots \ldots \ldots \ldots \ldots$

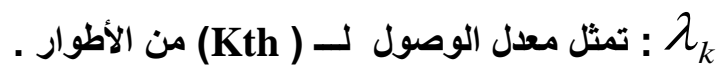

دالة الكثافة الاحتمالية

\section{Probability Density Function}

إن تحويل لابلاس لمجموع المتغيرات العثوائية المستقلة هو حاصل ضرب تحويلات لابلاس لدوال الكثافة

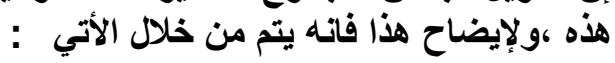

$$
\begin{aligned}
& L(s)=\frac{\lambda_{1}}{s+\lambda_{1}} \cdot \frac{\lambda_{2}}{s+\lambda_{2}} \ldots \ldots \ldots \ldots \ldots . . \frac{\lambda_{k}}{s+\lambda_{k}} \\
& L(s)=\prod_{k=1}^{k} \frac{\lambda_{k}}{s+\lambda_{k}} \\
& \lambda_{k} \neq \lambda_{k+1} \quad, \forall_{k}=1,2 \\
& K-1 \\
& \therefore L(s)=\prod_{k=1}^{K} \lambda_{k} \sum_{i=1}^{K} \frac{\left(s+\lambda_{i}\right)^{-1}}{\prod_{\substack{k=1 \\
k \neq i}}^{K} \lambda_{k}-\lambda_{i}}
\end{aligned}
$$

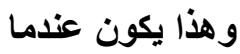

من تحويل لابلاس (؟ ـ r ) ) لتوزيع ايرلاتك العام نحصل على دالة الكثافة الاحتمالية

$$
a(t)=\prod_{k=1}^{K} \lambda_{k} \sum_{i=1}^{K} \frac{e^{-\lambda_{i} t}}{\prod_{\substack{k=1 \\ k \neq i}}^{K} \lambda_{k}-\lambda_{i}}
$$




\section{Parameters of Generalized Erlang Distribution}

معالم توزيع ايرلاتك العام

في هذه الفقرة سنتعرف على معالم التوزيع (الوسط الحسابي، التباين، ومعامل الاختلاف) في حالة

من المعادلة) ـ - ب ) لهذا التوزيع ، يتم حساب الوسط الحسابي والتباين للتوزيع نفسـه ، وهذا يتم

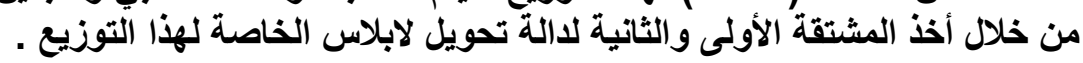

$\frac{d L(s)}{d s}=\prod_{k=1}^{K} \lambda_{k} \sum_{i=1}^{K} \frac{(-1)\left(s+\lambda_{i}\right)^{-2}}{\prod_{\substack{k=1 \\ k \neq i}}^{K} \lambda_{k}-\lambda_{i}}$

$\frac{d^{2} L(s)}{d s^{2}}=\prod_{k=1}^{K} \lambda_{k} \sum_{i=1}^{K} \frac{2\left(s+\lambda_{i}\right)^{-3}}{\prod_{\substack{k=1 \\ k \neq i}}^{K} \lambda_{k}-\lambda_{i}}$

$\left.\frac{-d L(s)}{d s}\right|_{s=0}=\prod_{k=1}^{K} \lambda_{k} \sum_{i=1}^{K} \frac{1}{\lambda^{2}{ }_{i} \prod_{\substack{i=1 \\ k \neq i}}^{K} \lambda_{k}-\lambda_{i}}=\sum_{k=1}^{K} \frac{1}{\lambda_{k}}$

$\left.\frac{d^{2} L(s)}{d s^{2}}\right|_{s=0}=\prod_{k=1}^{K} \lambda_{k} \sum_{i=1}^{K} \frac{2}{\lambda_{i}^{3} \prod_{\substack{k=1 \\ k \neq i}}^{K} \lambda_{k}-\lambda_{i}}$

$\bar{t}=\sum_{k=1}^{k} \frac{1}{\lambda_{k}}=$ mean $=\frac{K}{\lambda}$

$\sigma^{2}{ }_{t}=\prod_{k=1}^{K} \lambda_{k} \sum_{i=1}^{K} \frac{2}{\lambda_{i}^{3} \prod_{\substack{k=1 \\ k \neq i}}^{K} \lambda_{k}-\lambda_{i}}-\left[\sum_{k=1}^{K} \frac{1}{\lambda_{k}}\right]^{2}$

$\sigma^{2}{ }_{t}=\sum_{k=1}^{K} \frac{1}{\lambda^{2}{ }_{k}}+\left[\sum_{k=1}^{K} \frac{1}{\lambda_{k}}\right]^{2}-\left[\sum_{k=1}^{K} \frac{1}{\lambda_{k}}\right]^{2}$

$\therefore \sigma^{2}{ }_{t}=\sum_{k=1}^{K} \frac{1}{\lambda^{2}{ }_{k}}=$ Variance $=\frac{K}{\lambda^{2}}$ 
$C_{t}^{2}=\frac{\left.\frac{d^{2} L(s)}{d s^{2}}\right|_{s=0}}{\left[\left.\frac{d L(s)}{d s}\right|_{s=0}\right]^{2}}$

$C^{2}{ }_{t}=\frac{\sum_{k=1}^{K} \frac{1}{\lambda^{2}{ }_{k}}}{\left[\sum_{k=1}^{K} \frac{1}{\lambda_{k}}\right]^{2}}=\frac{1}{K}$

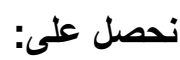

Specialized Erlang Distribution

ع - ا ثوزيع ايرلانك الخاص

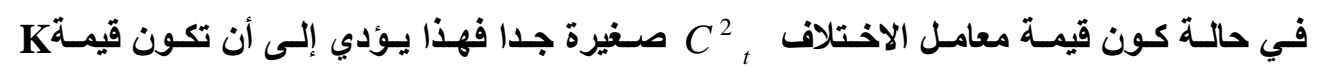

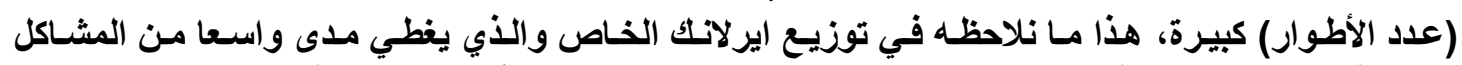

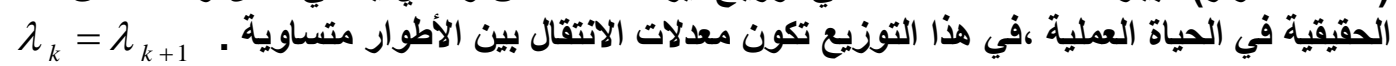

Probability Density Function

دالة الكثافة الاحتمالية

عندما تكون معدلات الانتقال بين الأطوار متساوية $\lambda_{k}=\lambda_{k+1}$ فان دالـة الكثافة الاحتمالية تكون كالأتي :

$L_{k}(s)=L_{k+1}(s)=\frac{K \lambda}{s+K \lambda} \quad, \forall_{k}=1,2, \ldots \ldots \ldots . ., K-1$

بما إن التوزيع الاحتمالي لكل طور من الأطوار يتبع التوزيع الآسي، من خلال دالة الكثافة الاحتمالية للتوزيع الآسي نحصل على انتى الآن $a_{k}(t)=a_{k+1}(t)=K \lambda e^{-K \lambda t} \quad, \forall{ }_{k}=1,2, \ldots \ldots \ldots \ldots-1$ (2-10)

$$
\text { من دالة تحويل لابلاس لتوزيع ايرلانك الخاص الموضحة من خلال المعادلة (r }
$$


ومن جذاول تحويل لابلاس نحصل على دالة الكثافة الاحتمالية الخاصة بهذا التوزيع :

$a(t)=\frac{(K \lambda)^{K} t^{K-1}}{(K-1) !} e^{-K \lambda t}$

\section{Parameters of Specialized Erlang Distribution}

معالم توزيع ايرلاتك الخاص

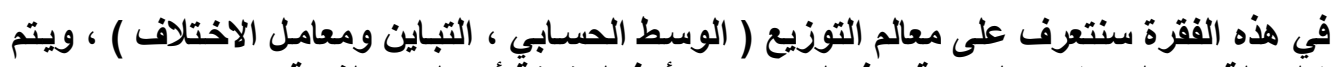

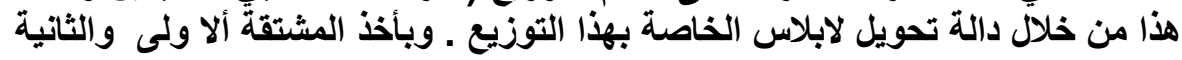

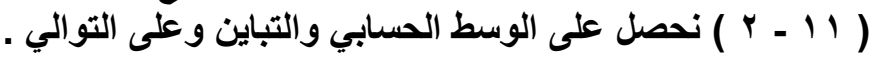

$\frac{-d L(s)}{d s}=-K(K \lambda)^{K}(s+K \lambda)^{-(K+1)}$

$\frac{d^{2} L(s)}{d s^{2}}=-K(-K-1)(K \lambda)^{K}(s+K \lambda)^{-(K+2)}$

$\bar{t}=K(K \lambda)^{K}(K \lambda)^{-K}(K \lambda)^{-1}$

$\bar{t}=\frac{1}{\lambda}=$ mean for each phase

$\sigma^{2}{ }_{t}=\frac{K(K+1)(K \lambda)^{K}}{(K \lambda)^{K+2}}-\frac{1}{\lambda^{2}}=\frac{1}{K \lambda^{2}}=$ Variance for each phase

$C_{t}^{2}=\frac{1 / K \lambda^{2}}{(1 / \lambda)^{2}}<1$

من خلال المعادلة العامة لمعامل الاختلاف ، نحصل على

تم الاعتماد في هذا البحث على نموذج صف انتظار ايرلاتك بمحطات خدمة متعددة وبسعة غير محددة $\left(M / E_{k} / c\right):(G D / \infty / \infty)$

معدل عدد الزبائن في صف الانتظار ( L q )

$L_{q}=\frac{\lambda^{2} /\left(k \mu^{2}\right)+\rho^{2}}{2(1-\rho)}=\frac{1+k}{2 k} \frac{\lambda^{2}}{\mu(\mu-\lambda)} \ldots \ldots \ldots(2-16)$ 


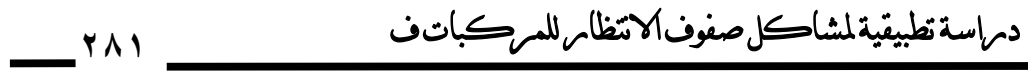

ي بعض محطات التعبيةتلمدينة غغداد

$W_{q}=\frac{1+k}{2 k} \frac{\lambda}{\mu(\mu-\lambda)} \ldots \ldots . .(2-17)$

معدل وقت الانتظار في صف الانتظار ( W

$$
\begin{aligned}
& w_{s}=w_{q}+\frac{1}{\mu} \quad \ldots \ldots \ldots \ldots(2-18) \\
& L_{s}=\lambda W_{s} \cdot \ldots \ldots \ldots \ldots(2-19)
\end{aligned}
$$

معدل وقت الانتظار في النظام ( ) م

معل عدد الزبائن في نظام ( L )

$\rho=\frac{\lambda}{c \mu} \quad \ldots \ldots \ldots . . .(2-20)$

معامل الاستخدام

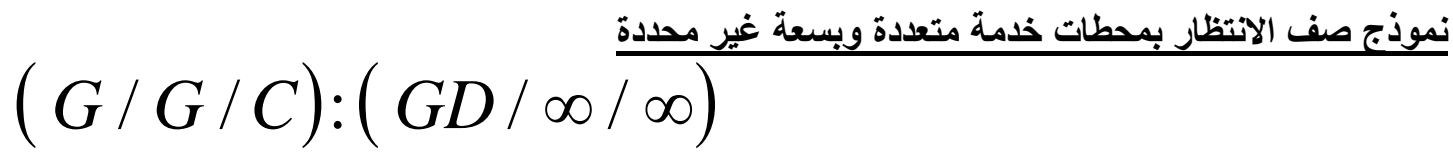

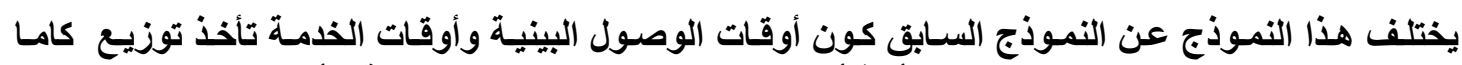
والتي قد تأخذ أي توزيع من التوزيعات الإحصائية كأن يكون توزيعات طبيعيا (General Distribution)

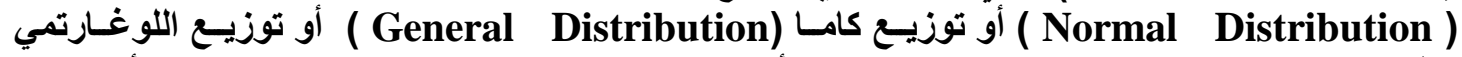

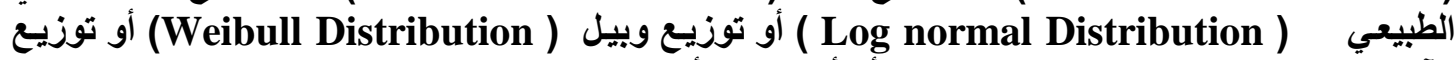

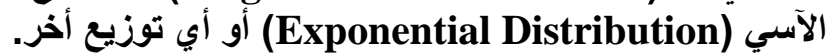

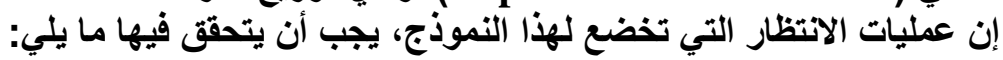

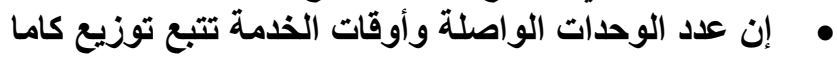

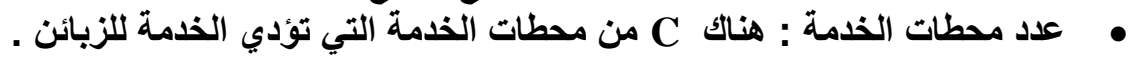
• • • • ليس هناك حدود لمدى استيعاب نظام الانتظار

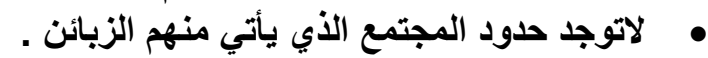

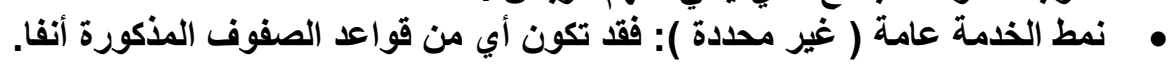

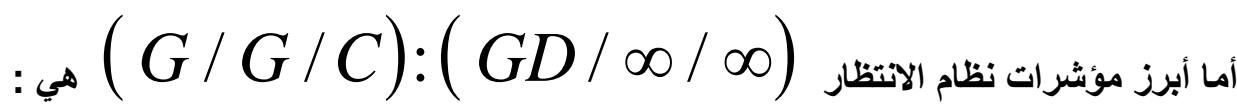




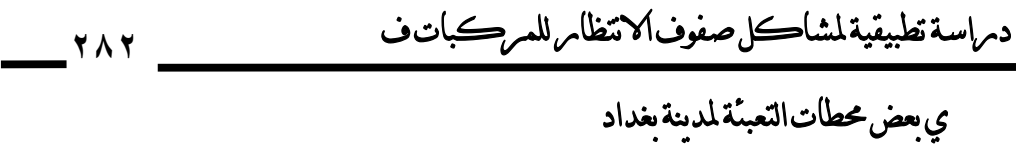

معدل عدد الزبائن في صف الانتظار ( L L )

$L_{q}=L_{q(M / M / C)} \cdot \frac{\mu^{2} V(t)+V\left(t^{\prime}\right) \lambda^{2}}{2}$

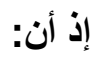

: : :

. تباين أوقات الوصول البينية .

$L_{q}=P_{0} \cdot \frac{\rho^{C+1}}{C ! . C} \cdot \frac{1}{\left(1-\frac{\rho}{C}\right)^{2}} \cdot \frac{\mu^{2} V(t)+V\left(t^{\prime}\right) \lambda^{2}}{2}$

$$
\begin{aligned}
& \text { - } \frac{\rho}{C}<1 \text { و إن } \\
& \text { معدل عدد الزبائن في نظام ( L s ) } \\
& L_{s}=L_{q}+\rho \\
& (2-23)
\end{aligned}
$$

معدل وقت الانتظار في صف الانتظار ( W

$$
W_{q}=\frac{L_{q}}{\lambda} \ldots \ldots(2-24)
$$

معدل وقت الانتار في النظام ( W

$$
W_{s}=W_{q}+\frac{1}{\mu} \ldots \ldots \ldots(2-25)
$$

احتمال وجود ( n ) من الزبائن في النظام ( P n )

$$
P_{(n)}= \begin{cases}P_{(n-1)} \cdot \frac{\rho}{n} & \text { for } n \leq C \\ P_{(n-1)} \cdot \frac{\rho}{C} & \text { for } n>c\end{cases}
$$




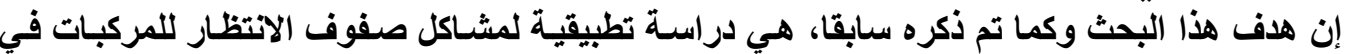

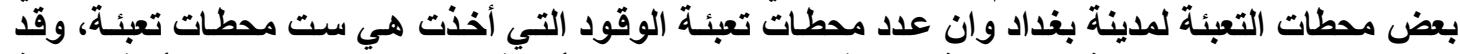

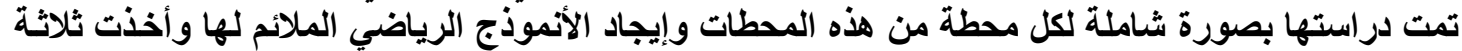

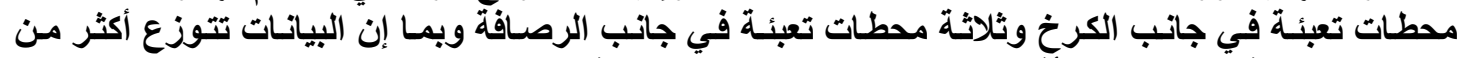

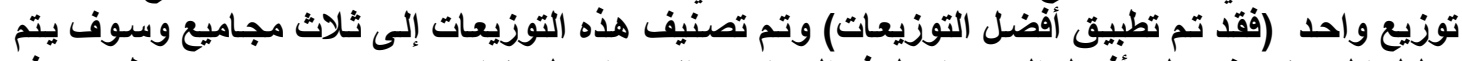

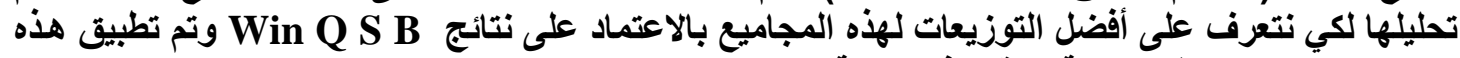

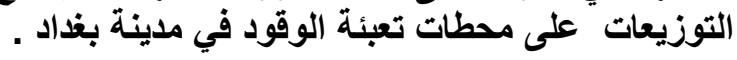

إلات ا و هذه المحطة تقع في جاتب الكرخ في منطقة حي الخضراء وتحتوي على 13 هوز وتم جمع بيانات منها لغ برض البحث

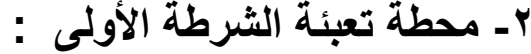

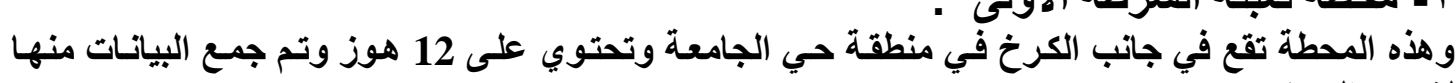

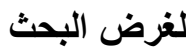

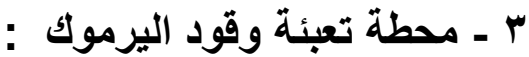
وهذه المحطة تقع في جاتب الكرخ في منطقة حي اليرموك وتحتوي على 14 هوز وتم جمع البيانات منها لغرض البحث ك - محطة تعبئة وقود المستتصرية : وهذه المحطة تقع في جاتب الرصافة في منطقة حي المستتصرية وتحتوي على 28 هوز وتم جمع البيانات

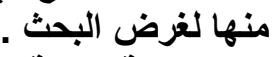

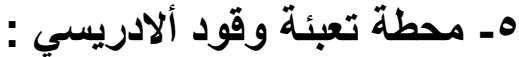
و هذه المحطة تقع في جانب الرصافةً بالقرب من ساحة المظفر وتحتوي على 22 هوز وتم جمع البياتات منها

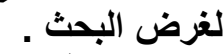
و - محطة تعبئة وقود الكيلاني : و هذه المحطة تقع في جانب الرصافة في منطقة الباب الثرقي وتحتوي على 28 هوز وتم جمع البيانات منها

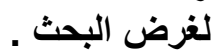

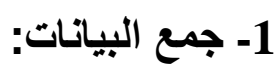

من خلال الزيارات والمراجعات للباحثة في محطات تعبئة الوقود اتضح عدم وجود بيانتات سابقة

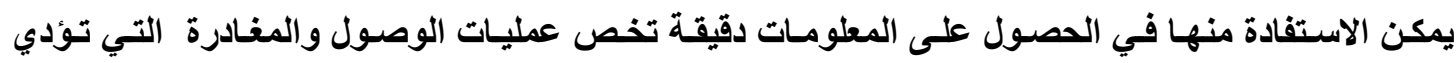

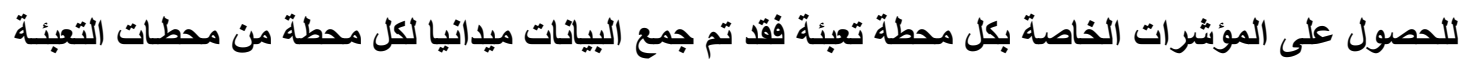

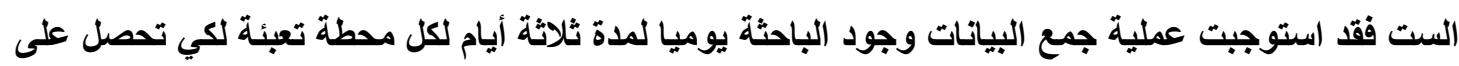

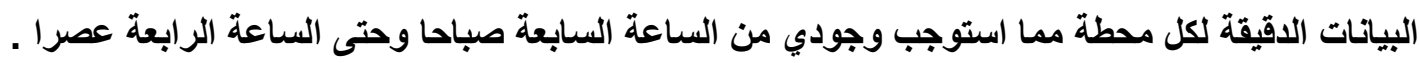




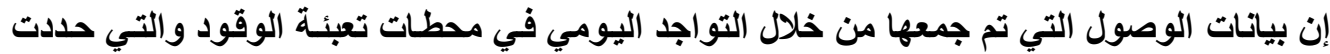

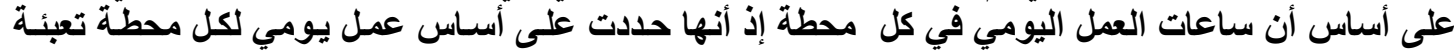

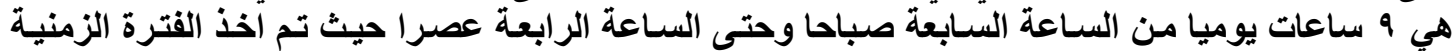

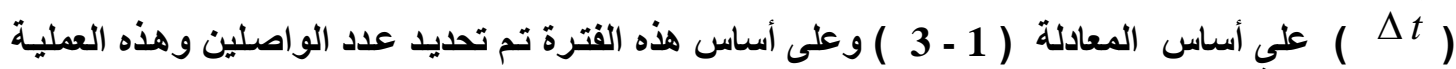
تمت لكل محطة على حدة المان

$$
\begin{aligned}
& \text { عدد ساعات العمل الفعلية } 60 \\
& \text { عدد السيار ات في النظام }
\end{aligned}
$$

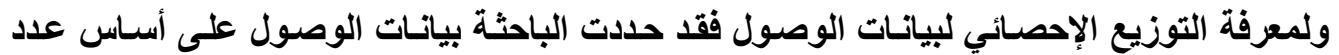

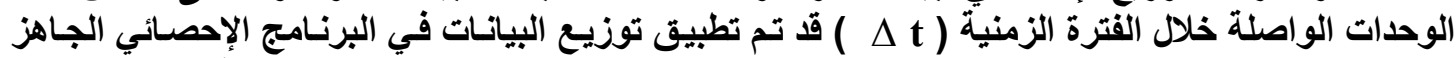
stat graphics أفضل توزيعين هما بو اسون وايرلانات إنكان

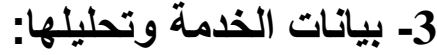

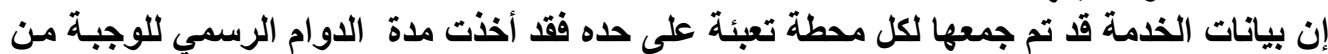

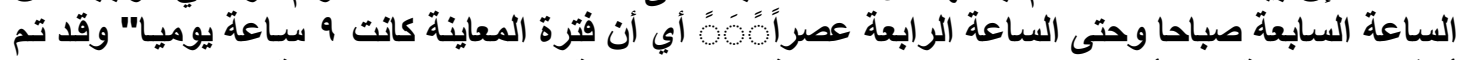

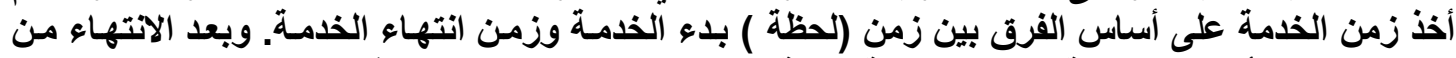

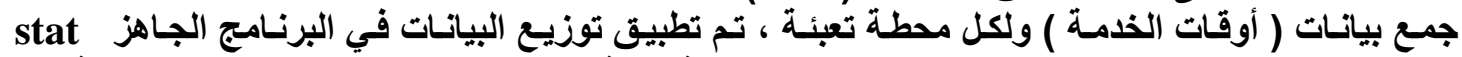
graphics تم اختيار أفضل توزيعين وهما الآسي وييل .

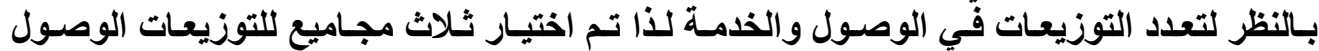
والخدمة لكي يتم المفاصلة بين هذه المجاميع ولئ والميع المجاميع هي:

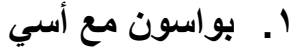

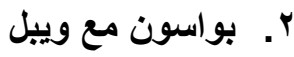

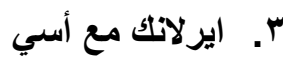


وقد كانت نتائج توزيع البيانات إحصائيا كالآتي :

محطة الخضر اء

\begin{tabular}{|c|c|c|c|}
\hline توزيع الوصول & وصول & توزيع الخدمة & خلمة \\
\hline Poisson & $\begin{array}{c}\text { Mean }=1.35479 \\
P \text { - Value }<0.01\end{array}$ & Exponential & $\begin{array}{l}\text { Mean }=7.43494 \\
P-\text { Value }<0.01\end{array}$ \\
\hline Poisson & $\begin{array}{l}\text { Mean }=1.35479 \\
P-\text { Value }<0.01\end{array}$ & Weibull & $\begin{array}{r}\text { Shape }=4.72145 \\
\text { Scale }=8.13559 \\
P-\text { Value }<0.01\end{array}$ \\
\hline Erlang & $\begin{array}{r}\text { Shape }=4 \\
\text { Scale }=2.95249 \\
P-\text { Value }<0.01\end{array}$ & Exponential & $\begin{array}{l}\text { Mean }=7.43494 \\
P-\text { Value }<0.01\end{array}$ \\
\hline
\end{tabular}

محطة اليرموك

\begin{tabular}{|c|c|c|c|}
\hline توزيع الوصول & وصول & توزيع الخدمة & خدمة \\
\hline Poisson & $\begin{array}{l}\text { Mean }=1.38793 \\
P-\text { Value }<0.01\end{array}$ & Exponential & $\begin{array}{l}\text { Mean }=6.58135 \\
P \text { - Value }<0.01\end{array}$ \\
\hline Poisson & $\begin{array}{l}\text { Mean }=1.38793 \\
P-\text { Value }<0.01\end{array}$ & Weibull & $\begin{array}{r}\text { Shape }=3.57395 \\
\text { Scale }=7.31843 \\
P-\text { Value }<0.01\end{array}$ \\
\hline Erlang & $\begin{array}{r}\text { Shape }=5 \\
\text { Scale }=3.60248 \\
\text { P }- \text { Value }<0.01\end{array}$ & Exponential & $\begin{array}{l}\text { Mean }=6.58135 \\
P-\text { Value }<0.01\end{array}$ \\
\hline
\end{tabular}


ي يعض مطات التعبّة لمدينة بغداد

محطة الشرطة الاولى

\begin{tabular}{|c|c|c|c|}
\hline توزيع الوصول & الوصول & توزيع الخدمة & الخدمة \\
\hline Poisson & $\begin{array}{l}\text { Mean }=1.14137 \\
P-\text { Value }<0.01\end{array}$ & Exponential & $\begin{array}{c}\text { Mean }=7.46354 \\
P-\text { Value }<0.01\end{array}$ \\
\hline Poisson & $\begin{array}{l}\text { Mean }=1.14137 \\
P-\text { Value }<0.01\end{array}$ & Weibull & $\begin{array}{l}\text { Shape }=6.24062 \\
\text { Scale }=8.02012 \\
P-\text { Value }<0.01\end{array}$ \\
\hline Erlang & $\begin{array}{r}\text { Shape }=14 \\
\text { Scale }=12.266 \\
P-\text { Value }<0.01\end{array}$ & Exponential & $\begin{array}{r}\text { Mean }=7.46354 \\
P-\text { Value }<0.01\end{array}$ \\
\hline
\end{tabular}

محطة الإدريسي

\begin{tabular}{|c|c|c|c|}
\hline توزيع الوصول & الوصول & توزيع الذذمة & الخدمة \\
\hline Poisson & $\begin{array}{l}\text { Mean }=1.50606 \\
P-\text { Value }<0.01\end{array}$ & Exponential & $\begin{array}{r}\text { Mean }=8.12368 \\
P-\text { Value }<0.01\end{array}$ \\
\hline Poisson & $\begin{array}{l}\text { Mean }=1.50606 \\
P-\text { Value }<0.01\end{array}$ & Weibull & $\begin{array}{l}\text { Shape }=7.75409 \\
\text { Scale }=8.63585 \\
P \text { - Value }<\mathbf{0 . 0 1}\end{array}$ \\
\hline Erlang & $\begin{array}{r}\text { Shape }=7.75409 \\
\text { Scale }=8.63585 \\
\text { P - Value }<0.01\end{array}$ & Exponential & $\begin{array}{r}\text { Mean }=8.12368 \\
P-\text { Value }<0.01\end{array}$ \\
\hline
\end{tabular}

محطة المستتصرية

\begin{tabular}{|c|c|c|c|}
\hline توزيع الوصول & الوصول & توزيع الخدمة & الخذمة \\
\hline Poisson & $\begin{array}{c}\text { Mean }=1.27941 \\
P-\text { Value }<0.01\end{array}$ & Exponential & $\begin{array}{r}\text { Mean }=6.92568 \\
P-\text { Value }<0.01\end{array}$ \\
\hline Poisson & $\begin{array}{c}\text { Mean }=1.27941 \\
P-\text { Value }<0.01\end{array}$ & Weibull & $\begin{array}{r}\text { Shape }=6.10442 \\
\text { Scale }=7.44983 \\
P-\text { Value }<0.01\end{array}$ \\
\hline Erlang & $\begin{array}{c}\text { Shape }=8 \\
\text { Scale }=6.25287 \\
\text { P }- \text { Value }<0.01\end{array}$ & Exponential & $\begin{array}{c}\text { Mean }=6.92568 \\
P-\text { Value }<0.01\end{array}$ \\
\hline
\end{tabular}

محطة الكيلاني

\begin{tabular}{|c|c|c|c|}
\hline توزيع الوصول & الوصول & توزيع الخدمة & الخدمة \\
\hline Poisson & $\begin{array}{r}\text { Mean }=1.26644 \\
P-\text { Value }<0.01\end{array}$ & Exponential & $\begin{array}{r}\text { Mean }=6.73017 \\
P-\text { Value }<0.01\end{array}$ \\
\hline Poisson & $\begin{array}{r}\text { Mean }=1.26644 \\
P-\text { Value }<0.01\end{array}$ & Weibull & $\begin{array}{r}\text { Shape }=4.30869 \\
\text { Scale }=7.3996 \\
P-\text { Value }<0.01\end{array}$ \\
\hline Erlang & $\begin{array}{r}\text { Shape }=4.30869 \\
\text { Scale }=7.3996 \\
P \text { - Value }<0.01 \\
\end{array}$ & Exponential & $\begin{array}{r}\text { Mean }=6.73017 \\
P-\text { Value }<0.01\end{array}$ \\
\hline
\end{tabular}




\begin{tabular}{|c|c|c|c|c|c|c|}
\hline المحطة & عدد الوحدات الخدمة & التوزيع & $\mathbf{L}_{\mathrm{s}}$ & $\mathbf{L}_{q}$ & $\mathbf{W}_{\mathrm{s}}$ & $\mathbf{W}_{\mathbf{q}}$ \\
\hline \multirow{3}{*}{ الخضراء } & 13 & بواسون مع اسبي & 5 & 0.0030 & 7.4390 & 0.0040 \\
\hline & 13 & بواسون مع وييل & 5 & 0.0014 & 7.4469 & 0.0019 \\
\hline & 13 & ايرلانك مع أسي & 4 & 0.130196 & 0.330000 & 0.195499 \\
\hline \multirow[t]{3}{*}{ اليرموك } & 14 & بواسون مع اسبي & 5 & 0.0002 & 6.5816 & 0.0003 \\
\hline & 14 & بواسون مع ويبل & 5 & 0.0001 & 6.5922 & 0.0001 \\
\hline & 14 & ايرلانك مع أسي & 5 & 0.282205 & 0.280208 & 0.128264 \\
\hline \multirow[t]{3}{*}{ الثرطة الأولى } & 12 & بواسون مع اسي & 7 & 0.0453 & 7.5152 & 0.057 \\
\hline & 12 & بواسون مع وييل & 7 & 0.0218 & 7.4814 & 0.0249 \\
\hline & 12 & ايرلانك مع أسي & 36 & 4 & 0.209857 & 0.0758726 \\
\hline \multirow[t]{9}{*}{ الإدريسي } & 22 & بواسون مع اسي & 5 & 0.0000 & 8.1237 & 0.0000 \\
\hline & 22 & بواسون مع ويبل & 5 & 0.0000 & 8.1203 & 0.0000 \\
\hline & 22 & ايرلانك مع أسي & 13 & 1 & 0.196327 & 0.0732301 \\
\hline & 12 & بواسون مع اسي & 5 & 0.0071 & 8.1344 & 0.0170 \\
\hline & 12 & بواسون مع ويبل & 5 & 0.0029 & 8.1247 & 0.0044 \\
\hline & 12 & ايرلانك مع أسي & 13 & 2.37399 & 0.198980 & $\mathbf{0 . 0 7 5 8 8 3 3}$ \\
\hline & 10 & بواسون مع اسبي & 5 & 0.0548 & 8.2062 & 0.0825 \\
\hline & 10 & بواسون مع وييل & 5 & 0.0226 & 8.1543 & 0.0340 \\
\hline & 10 & ايرلانك مع أسي & 13 & 5 & 0.200148 & 0.0770508 \\
\hline \multirow[t]{9}{*}{ المستنصرية } & 28 & بواسون مع اسبي & 5 & 0.0000 & 6.9257 & 0.0000 \\
\hline & 28 & بواسون مع وييل & 5 & 0.0000 & 6.9179 & 0.0000 \\
\hline & 28 & ايرلانك مع أسي & 12 & 1 & 0.231180 & 0.0867895 \\
\hline & 18 & بواسون مع اسي & 5 & 0.0000 & 6.9257 & 0.0000 \\
\hline & 18 & بواسون مع ويبل & 5 & 0.0000 & 6.9179 & 0.0000 \\
\hline & 18 & ايرلانك مع أسي & 12 & $\mathbf{1}$ & 0.232842 & 0.0884521 \\
\hline & 10 & بواسون مع اسبي & 5 & 0.0603 & 7.0028 & 0.0772 \\
\hline & 10 & بواسون مع وييل & 5 & 0.0274 & 6.9531 & 0.0351 \\
\hline & 10 & ايرلانك مع أسي & 12 & 3 & 0.236567 & 0.0921764 \\
\hline \multirow[t]{9}{*}{ الكيلاني } & 28 & بواسون مع اسبي & 5 & 0.0000 & 6.7302 & 0.0000 \\
\hline & 28 & بواسون مع وييل & 5 & 0.0000 & 6.7357 & 0.0000 \\
\hline & 28 & ايرلاتك مع أسي & 8 & 0.440839 & 0.246119 & 0.0975355 \\
\hline & 18 & بواسون مع اسي & 5 & 0.0000 & 6.7302 & 0.0000 \\
\hline & 18 & بواسون مع ويبل & 5 & 0.0000 & 6.7357 & 0.0000 \\
\hline & 18 & ايرلانك مع أسي & 8 & 1 & 0.247988 & 0.0994029 \\
\hline & 10 & بواسون مع اسبي & 5 & 0.0524 & 6.7965 & 0.0663 \\
\hline & 10 & بواسون مع وييل & 5 & 0.0253 & 6.7677 & 0.0320 \\
\hline & 10 & ايرلانك مع أسي & 8 & 1 & 0.252173 & 0.103588 \\
\hline
\end{tabular}


وظهر لنا بعد تطبيق البرنامج الجاهز Win QSB إن أفضل توزيع في محطة الخضراء هو توزيع إنايع

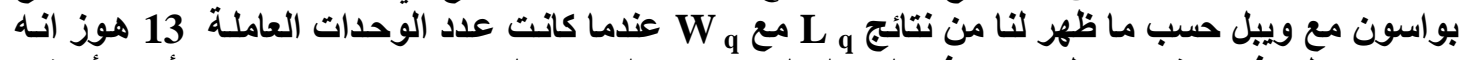

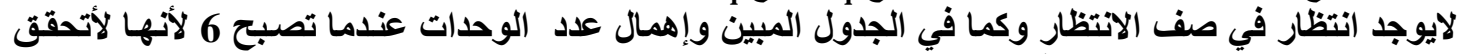
الهاف المطلوب وهو تقابليل كلفة وزيادة الإرباح.

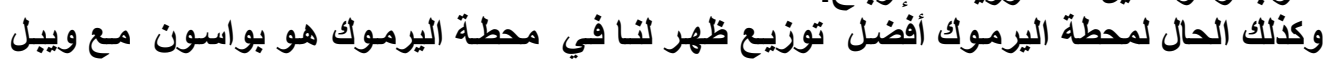

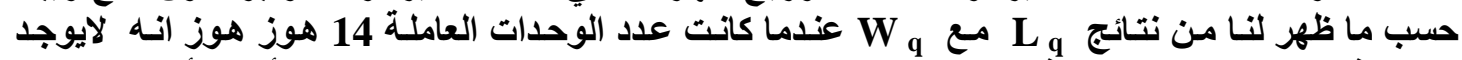
انتظار في صف الانتظار وكما في الجدول المبين وإهمال عدد الوحدات عندما تصبح 5 لأنها لأتحقق الهدف لإنف

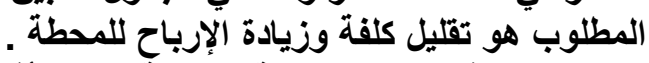

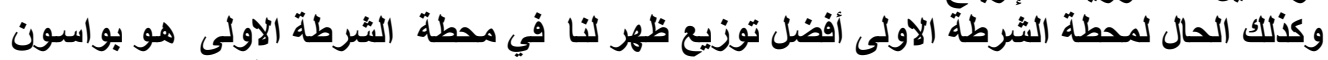

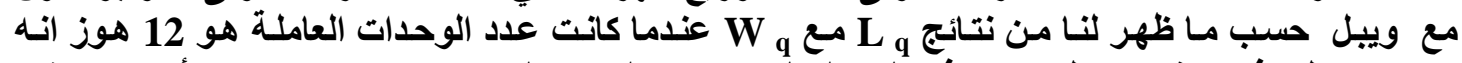

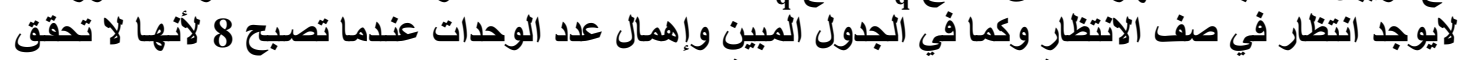

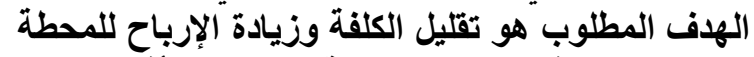

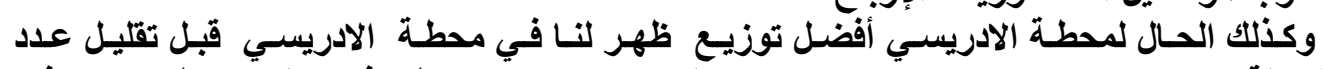

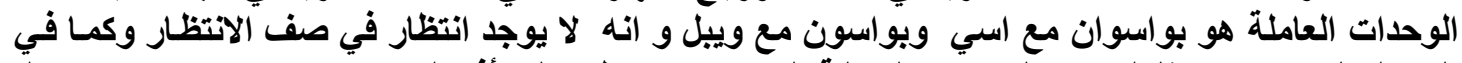

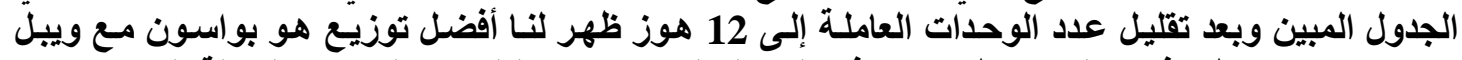

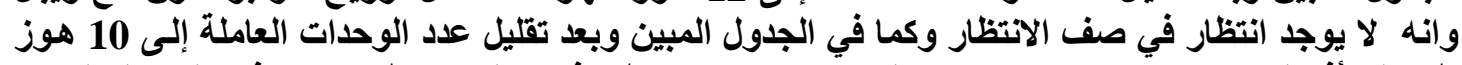

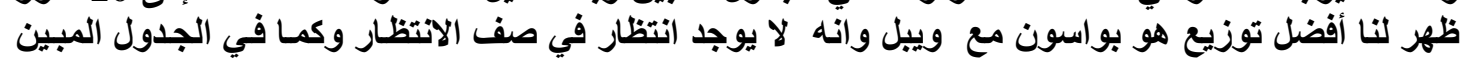

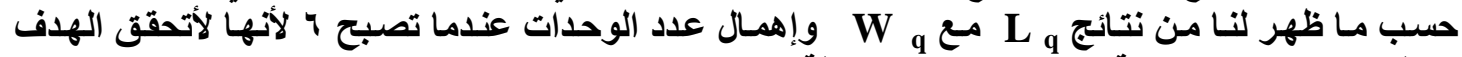

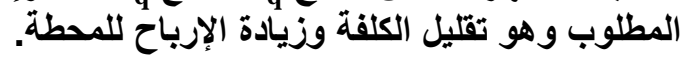

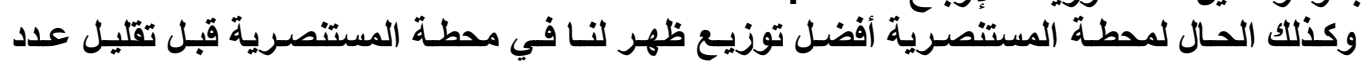

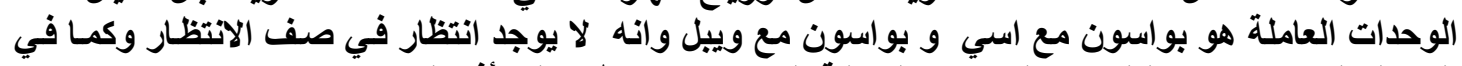

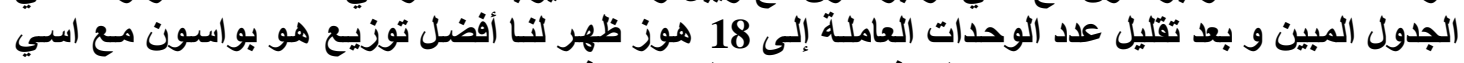

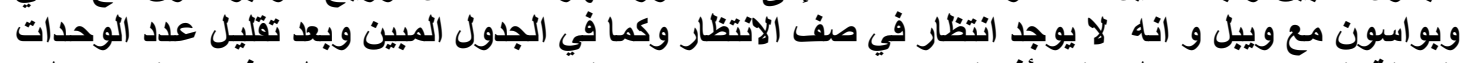

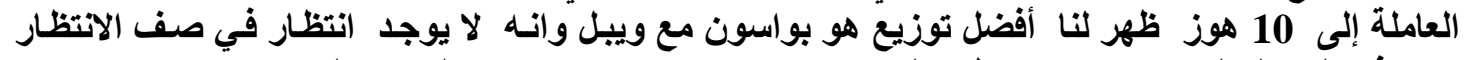

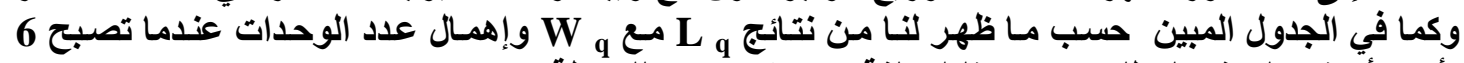

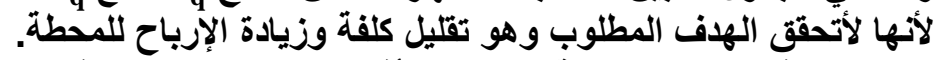

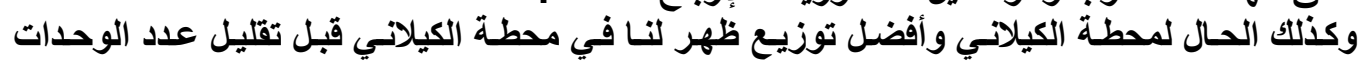

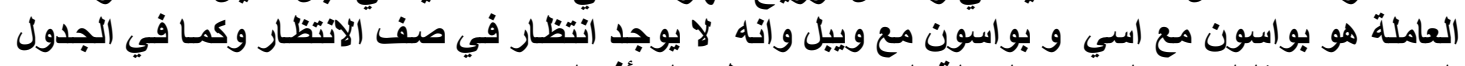

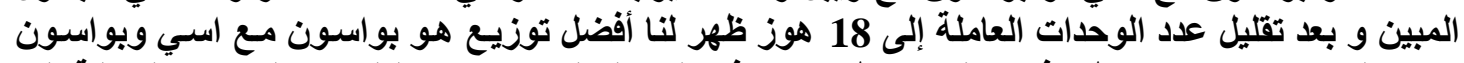

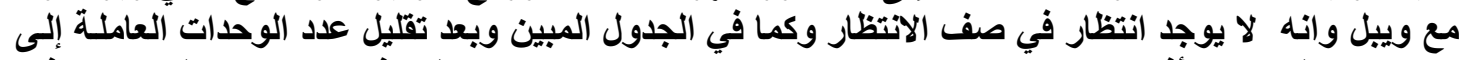

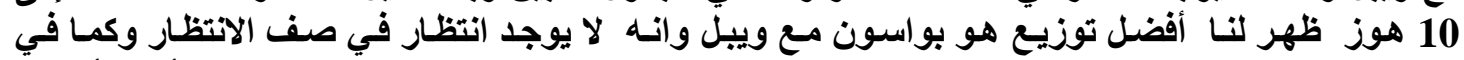

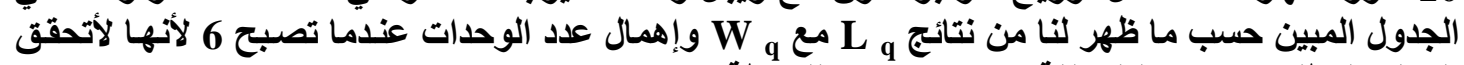

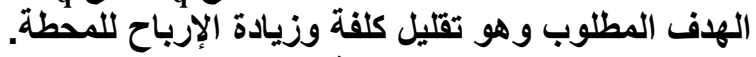

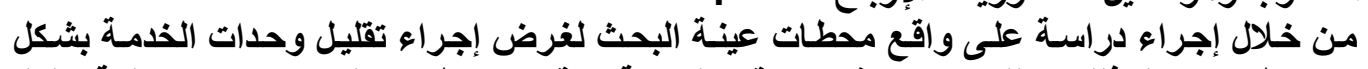

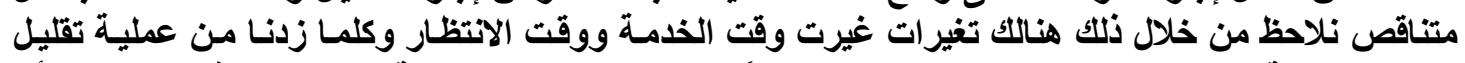

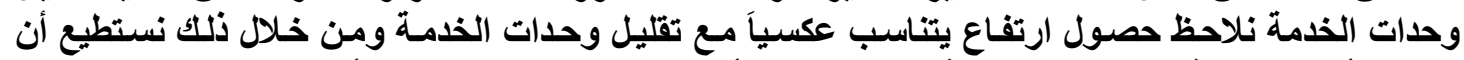

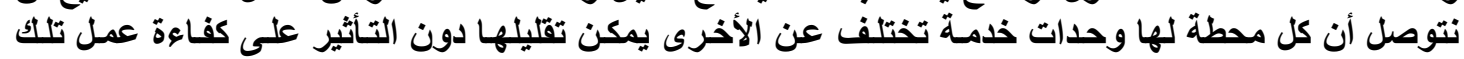

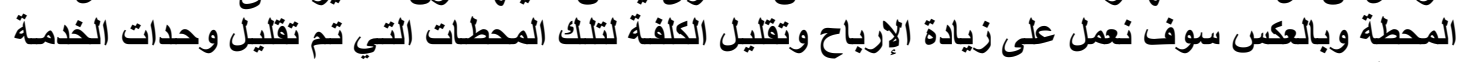
العاملة فيها كما في الجدول.

نلاحظ في محطات الصغيرة لايكون فيها تقليل في عدد الوحدات العاملة حتى لايؤثر على عمل المحطة بينما

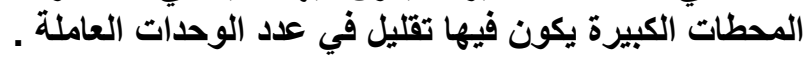




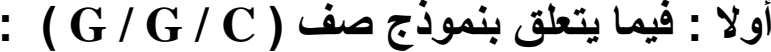

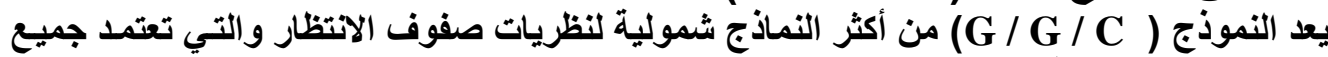

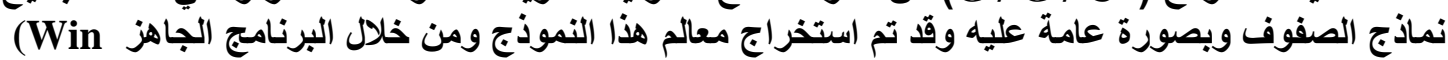

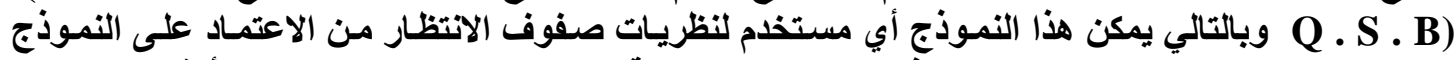

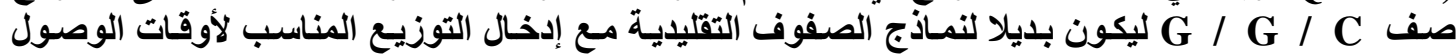
والخدمة وكنلك إدخال عدد محطات الخدمة.

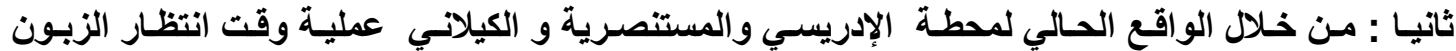

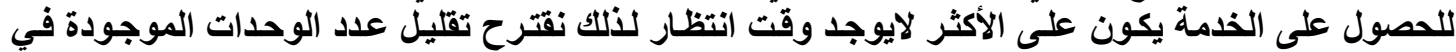

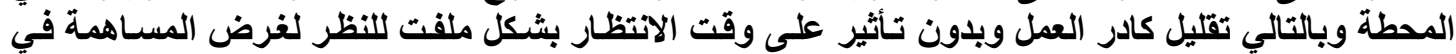
تقليل تكلفة المحطة وزيادة الإرباح .

ثالثاً : نلاحظ من خلال مجاميع التوزيعات بان المحطات ذوات عدات الوحدات العاملة القليلة مثل محطات الكرخ

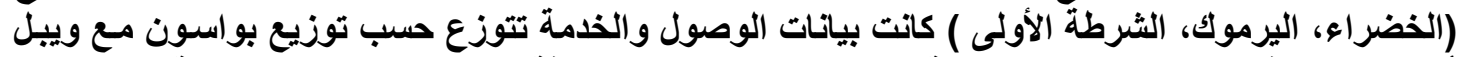

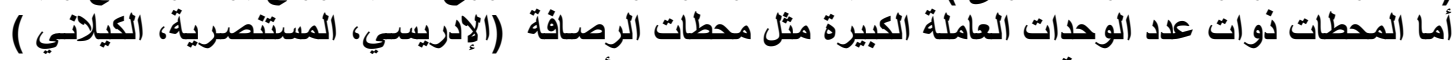

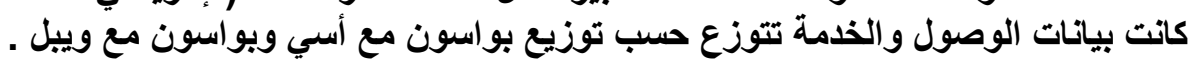

رابعاً نلاحظ في المحطات ذوات عدد الوحدات العاملة القليلة كما في محطات ( الخضراء واليرموك والثئ والثرطة

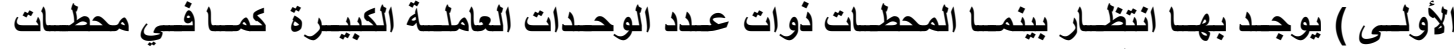

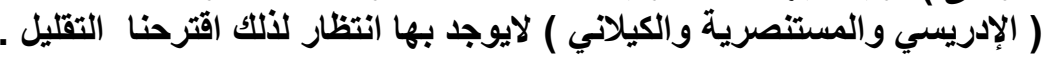

يجب أن تتو افر هنالك قاعدة كاملة للبيانات في كل محطة يدون فيها ما يلي، ليتسنى للباحثين الحصول

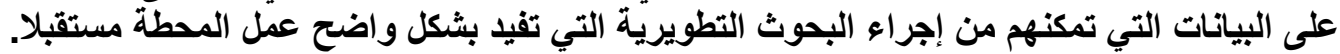

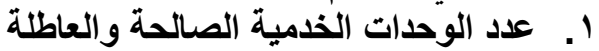

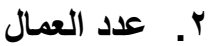
r. ب. كميات الوقود المتوفرة لكل محطة

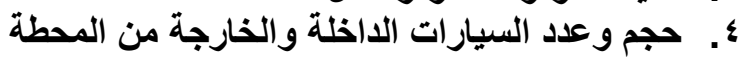

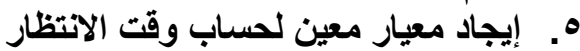
T. كلفة المضخة V. Vلفة العامل 


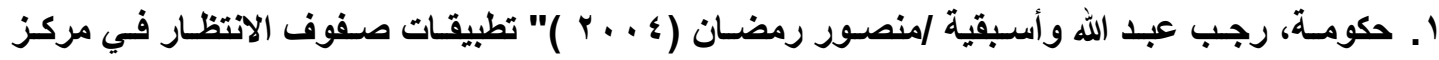

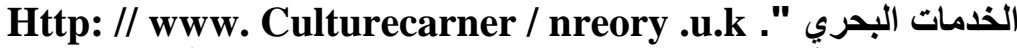

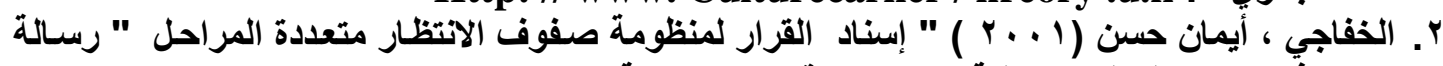

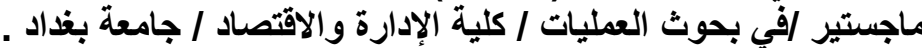

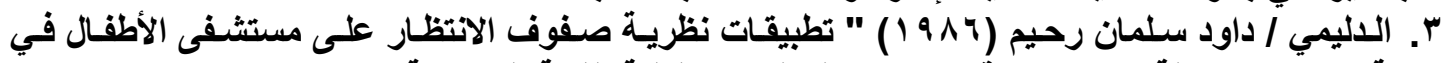

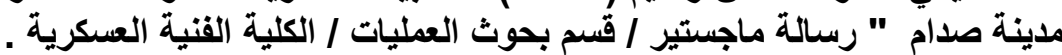

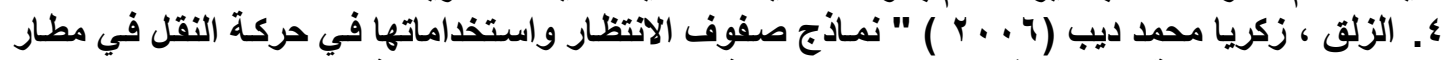

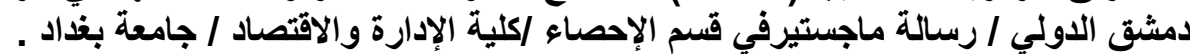

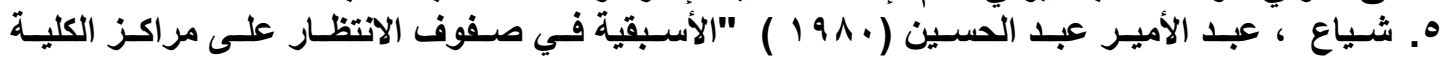

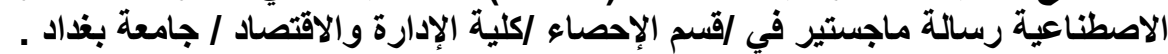

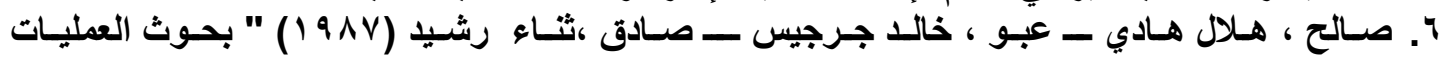

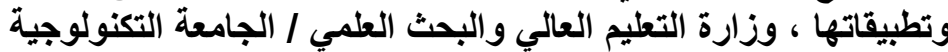

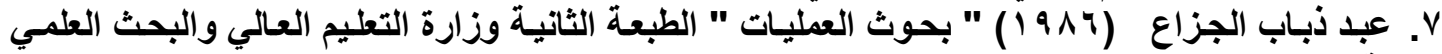

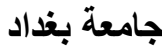

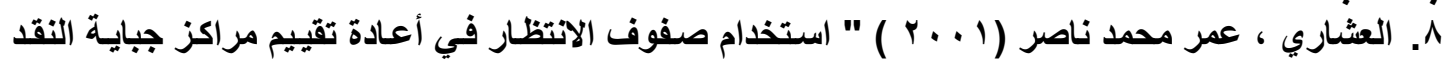

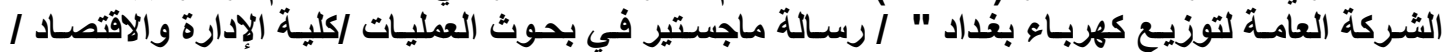

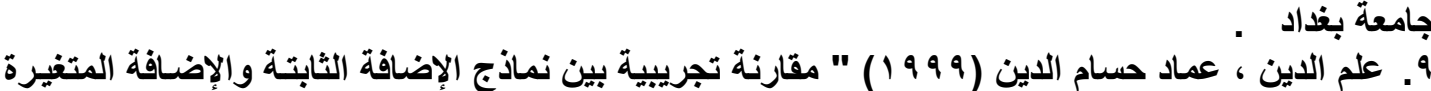

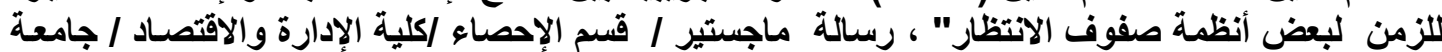

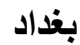

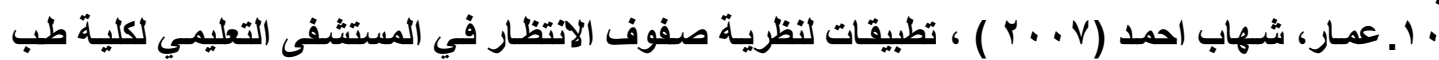

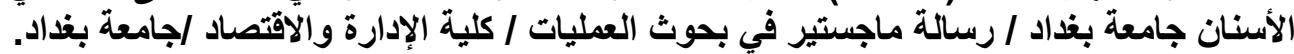

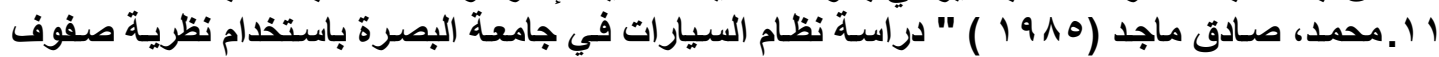

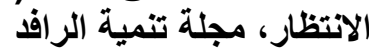

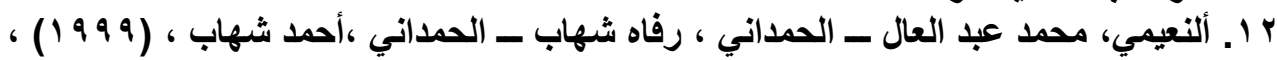


13 - A matt R, Depalma A., (2000), "Queuing Process" Tin Bergen Institute Discussion Papers for $\mathrm{m}$ Tin Bergen Institute.

14 - Bell, Colin E., (1985)," on competition To Join A Simple Queuing system Before the Facility open ", Management Science Vol.31,No,3 .

15- Cob ham, A. (1954) "Priority Assignment In waiting Line problem". Oper Res. 2, 70 - 76, Correction Ibid. 3,547.

16 - Crommelin, C.D. (1932). "Mathematisches unbar Die Erwotung vor Einem offen Lichen schater".Mat. Sbronik. 39, 73 - 84.

17- Dakheel, F. I (1990), "A decision Support system for Single stage Markovian Queuing system, "Ph. D.thesis, University of Brad Ford.

18-Dakheel , F.I (1996) , " An Approach For Determing the Analytical solution the Machine Interference model $\left(\mathbf{E}_{\mathbf{k}} / \mathbf{E}_{\mathrm{L}} / \mathbf{m} / \mathbf{N}\right) "$ college of Education , Al Mustansiriayah university .

19 - Erlang, A.K. (1909). "The theory of probabilities and Telephone conversation." Nyt Tidsskrift Matematik., B.20.33-39.

20 - Erlang, A.K. (1917). "Solution of some problems in the theory of probabilities of significance In Automatic Exchanges ". Electroteknikeren (Danish ) 13- 5 - 13

21 - Evans, James R. (1993) "Applied production and operations Management ", $\left(4^{\text {th }}\right.$ ed) .west publishing company.

22 - Fry, T. C. (1928). "Probability and Its Engineering uses" Princeton N. J.: Van Non Strand.

23 - Gross, D and Harris, (1974), "Fundamentals of Queuing Theory", "New York :John wiley and sons.

24 -Galliher, H.P., and wheeler, R. C. (1958). "Non stationary Queuing probabilities for Landing Congestion of Aircraft". Oper. Res. 6, $264-275$.

25 -Hiller, Frederick s. Lieberman, (1990) $\left(3^{\text {rd }}\right.$ edition), "Introduction to operations Research", McGraw- Hill Inc

26 - Hall, Randolph W., (1989), "Expected performance of a Queuing System with Ancillary Activities", Operational Research Society Ltd.

27 - Hillier / Lieberman, (2001) $\left(7^{\text {th }}\right.$, edition). Introduction to Operations Research .873 $-875$.

-John h. and D. (1999) "Numerical Methods using $r \wedge$

Mat lab $3^{\text {rd }}$ Edition, Prentice - Hall .Inc Simon and Schuster A Viacom Company.

29 - Klein rock, Leonard (1976), "Queuing systems Val, John Wiley and Sons, Inc

30 - Khintchine, A.Y. (1932). "Mathematisches unbar Die Erwotung vor Einem offen Lichen schater". Mat.sbronik. 39, 73 - 84.

31 - Kolmogorov, A.N (1931). "SurLa problem D' Attente."Mat. Sbronik. 8, 101 - 106. 


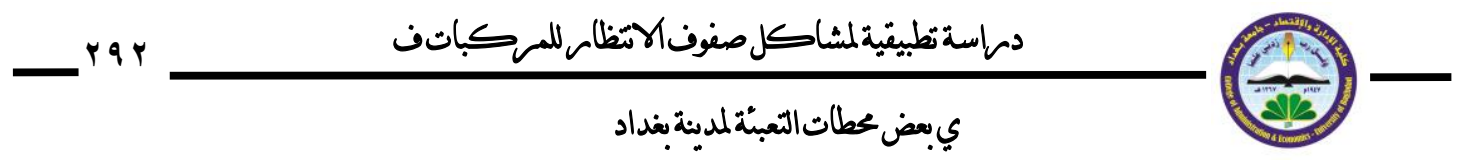

32 - Lawrence, John A - Pasternak Barry Alan, (1998), "Applied Management science. John Wiley and sons.

33 - Molina, E.C. (1927). "Application of the theory of probability to Telephone Trucking problems." Bell system Tech .J.6, 461-494

34 - Mad Christian N., (1988), "A Closed Queuing Maintenance Network with Two R Epicenters, "operational Research Society Ltd.

35- Pollaczek, F. (1932). "Losing Seines Geometries chem. Wahrs cheinlich ". Math. Z. 35, 230 - 278.

36- Philips, Don. T- Ravindran , A-Solberg, James (1987) "Operations Research Principles and practice " $\left(2^{\text {nd }}\right.$ ed.), John Wiley and sons, New York.

37 - Palm, C. (1938). "Analysis of the Erlang Traffic Formula for Busy - signal Arrange mens." Ericsson Tech. 6, 36 - 58.

38 - Rosen shines, M. (1967) "Queues with state - Dependent Service Times ". Trans p. Res. 1, 97, 104.

39 - Taka Cs, Lagos. (1962). "Theory of Queuing". Oxford university of Press.

40 - Taha, Hamdy A. (1997) "Operations Research an Introduction", (16 edition), Prentice - Hall. Inc Simon and Schuster A Viacom Company.

41 - Taha, H. (1982, 2003). "Operations Research". Macmillan publishing Co. Inc New York. 